\title{
Semi-Supervised Near-Miss Fall Detection for Ironworkers with a Wearable
} \section{Inertial Measurement Unit}

\author{
Kanghyeok Yang ${ }^{1}$, Changbum R. Ahn ${ }^{2 *}$, Mehmet C. Vuran ${ }^{3}$, and Sepideh S. Aria ${ }^{4}$
}

$1 \mathrm{PhD}$ Student, Charles Durham School of Architectural Engineering and Construction, University of Nebraska-Lincoln, W113 Nebraska Hall, Lincoln, NE 68588; PH (402) 472-5631; kyang11 @ unl.edu 2 Assistant Professor, Charles Durham School of Architectural Engineering and Construction, University of Nebraska-Lincoln, W113 Nebraska Hall, Lincoln, NE 68588, PH (402) 472-7431, cahn2@unl.edu

3 Associate Professor, Department of Computer Science and Engineering, University of NebraskaLincoln, 214 Schorr Center, Lincoln, NE 68588, PH (402) 472-5019, mcvuran@cse.unl.edu 4 Master Student, Department of Computer Science and Engineering, University of Nebraska-Lincoln, 256 Avery Hall Lincoln, NE 68588,PH (402) 472-2401, aria.sepi@ gmail.com 12

\section{Abstract}

Accidental falls (slips, trips, and falls from height) are the leading cause of occupational death and injury in construction. As a proactive accident-prevention measure, near-miss can provide valuable data about the causes of accidents, but collecting near miss information is challenging because current data collection systems can largely be affected by retrospective and qualitative decisions of individual workers. In this context, this study aims to develop a method that can automatically detect and document near-miss falls based upon a worker's kinematic data captured from wearable inertial measurement units (WIMUs). A semi-supervised learning algorithm (i.e., One-Class Support Vector Machine) was implemented for detecting the near-miss falls in this study. Two experiments were conducted for collecting the near-miss falls of ironworkers and these data were used to test developed near-miss fall detection approach. This WIMU-based approach will help identify ironworker near-miss falls without disrupting jobsite work and can help prevent fall accidents.

Keywords: Ironworker, Near-miss fall, Fall Accident, Machine Learning, Anomaly

\section{Detection}




\section{Introduction}

The construction industry is considered one of the most dangerous industries in the United States.

30 Among construction-related accidents, fall accidents are one of the leading causes of fatalities and

31 account for more than $30 \%$ of fatal accidents during recent decades [1]. Ironworkers are exceptionally susceptible to fall accidents and face the highest lifetime risk of fatal injuries among construction trades [2]. According to Beavers [3], between 2000 and 2005, more than $75 \%$ of fatal ironwork accidents were caused by fall accidents. Such high fatality rates among ironworkers are rooted in various factors: 1) most of the time, ironworkers are working on narrow surfaces of structural steel beams installed at high elevations and are thereby exposed to numerous open edges, 2) ironwork construction consists of physically demanding tasks such as handling heavy steel materials (e.g., beams, columns, and steel plate) and equipment for steel erection. These work characteristics contribute to the risks that ironworkers face in their daily tasks.

Due to the high risk of fall accidents among ironwork erection projects in general construction, the Occupational Safety and Health Organization (OSHA) requires the use of fall protection measures such as guardrails, safety nets, and personal protective equipment to protect workers when working at 43 elevation. The current safety measures for fall accidents are classified as active/primary protection 44 measures (e.g., guardrails and covers), which physically prevent the occurrence of falls, and 45 passive/secondary protection measures (e.g., personal fall-arrest systems, safety nets), which help to prevent or minimize injury from falls $[4,5]$. While passive/secondary protection measures are generally

47 employed for ironworkers, the use of active/primary fall-protection measures is limited due to the constraints of ironworkers' working environments. This shortcoming faces particular criticism since current fall-protection measures emphasize reducing the severity of an injury rather than proactively 
51 prevent the occurrence of fall itself during ironwork. Even though the proper use of PFAS can save the 52 life of workers who fall after losing their balance, being suspended in a harness may result in a suspension 53 trauma, orthostatic intolerance, or other, more severe injuries [7]. Moreover, many workers still get 54 injured due to using incomplete or inadequate fall protection devices [8].

In pursuit of a proactive approach for preventing accidents (including falls), researchers have turned their attention to collecting and utilizing accident leading indicators $[9,10]$. Leading indicators are conditions, events, or measures that are valuable in predicting the future occurrence of undesirable events, including accidents, incidents, or near misses [9]. Leading indicators are designed to monitor the safety process by identifying gaps between the current environment and recommended settings $[9,11]$. The knowledge identified through this monitoring is then used to decrease the possibility of injury occurrence

61 [11]. Thus, such indicators are associated with proactive approaches seeking to identify, assess, and eliminate a related risk [10]. A near miss is one such accident leading indicator $[11,12]$ and is defined as an event that causes no damage or loss at the time of occurrence but could have materialized into an actual accident in a slightly different environment [13]. Near misses have been widely used to reduce the likelihood of future accidents in diverse industries (e.g., chemical, airline, nuclear, railroad, medicine, and can help identify hazardous elements and vulnerable workers. This knowledge can also provide opportunities to eliminate such hazardous conditions at the job site or to alert possible victims that they need to change their behavior before an accident. For this reason, identifying and collecting near misses is an important step in implementing proactive accident-prevention measures. of near misses and introduced different applications of near-miss data [12,14,18-20]. In such cases, the collection of near-miss data mainly depends on a self-reporting system of workers. However, collecting 
qualitative and retrospective decisions based upon the perceived attitudes of individual workers, such as, fear of discipline, acceptance of risk, and inconsistent perception of near-miss falls [18]. One of the available methods for collecting quantitative near-miss data is using a technology solution — such as Ultrasound or RFID — to acquire real-time information about workers' exposure to known jobsite hazards [21]. However, such a proximity-based approach is not sufficient to capture near misses that are caused by unknown hazards or not triggered by physical hazards. Alternatively, one promising technology is WIMUs, which can document subtle body movements (i.e., acceleration, angular velocity) with a 3-axis accelerometer, gyroscope and magnetometer. In previous studies, WIMUs were widely used in fall 84 detection [22-26], activity recognition [27-33], and gait analysis [34-36]. Due to its small size, a WIMU developed a near-miss falls detection approach.

\section{Research Background}

Many previous studies have focused on identifying fall accident risk factors to increase worker

91 safety in construction. Chi [4] investigated accident patterns of previous fatal fall accidents and proposed

92 accident-prevention measures for each type of accidental scenario. Huang [8] identified factors related to 93 fall accidents (i.e., cause of accident, fall height, and accident-related elements) based on accident records 94 from OSHA, and Cattledge [37] studied injury records of nonfatal falls and identified problems with 95 current fall accident-prevention measures. As a proactive approach to accident prevention, Wu [12] 96 developed a systematic model for identifying near-miss information from on-going project using 97 knowledge from historical accident database. Wu deduced knowledge from previous accidents and 98 applied this knowledge to an ongoing construction project with a real-time near-miss tracking and 99 reporting system that used RFID technology. Cambraia [19] provided near-miss guidelines utilizing near100 miss information for accident prevention. Cambraia collected near-miss data from construction projects, 
analyzed the risks of identified near misses, and suggested recommendations for implementing a near-

102 miss system. Finally, Navon [38] introduced an automated fall-hazard monitoring system for construction

103 sites. This study identified the risks of fall accidents in activities included in project schedules and

104 proposed a guardrail-installation measures to prevent fall accidents. Also, this study monitored the status

105 of guardrail installation (e.g., missing or incomplete) through wireless communication to enhance

106 worksite safety.

However, previous studies have only focused on investigating previous accidents to derive general recommendations $[4,8,37]$ or on identifying potential accidents using previously known hazards and locations $[12,19,38]$. Moreover, previous techniques have failed to consider individual workers, who are actually at risk of accidents while working on construction sites. In particular, proximity-based

111 systems [12,38] require substantial resources to prevent ironworker fall accidents since this population

112 almost always works near open edges on narrow-surface steel beams. This exposure problem is 113 compounded by the fact that ironworkers have only a small surface space to recover from even a small 114 degree of balance loss, and current proximity based systems do not address this issue in real time. These 115 shortcomings of proximity based systems emphasize the current challenge in implementing fall116 prevention for ironworkers and highlight the reasons this population is still at the high risk of fall 117 accidents in construction. Information technology-based construction site data (e.g., about workers, work environment) 119 collection measures assist in increasing the ability to store, retrieve, and manipulate data during the 120 construction process [39]. As a possible alternative to capturing near-miss data, a WIMU, which includes 121 an accelerometer, gyroscope, and magnetometer, can robustly document and wirelessly communicate 122 human movement data. This advantage has led to the use of WIMUs for fall detection to increase the 123 safety of patients and workers [22-26]. In biomedical studies, attaching accelerometers to the body is a 124 widely used approach for detecting fall accidents, especially for the elderly [22,24]. Automated detection 125 of fall accidents using wearable sensors has been considered a promising method for protecting the 
126 elderly or people with a disability from an unidentified fall risk. In previous biomedical studies [22-24],

127 the key research objective focused on detecting fall accident situations or accident-related conditions such

128 as accident location, severity of injury, or injured body parts. These previous studies approached fall

129 accident detection by detecting strong accelerometer signals from when a subject actually fell to the

130 ground rather than by identifying or monitoring dangerous movements (e.g., loss of balance) that reveal

131 informative data about future or potential fall accidents. This limited focus is problematic for translating

132 biomedical research to ironworkers since according to Beavers [3], loss of balance (LOB) is one of the

133 major proximal causes of fall accidents for ironworkers, and LOB is cited as contributing to most

134 unintentional falls even in spite of the lack of a quantitative definition [40].

There are a few studies that attempt to detect near-miss falls_-sometimes called "near-falls"-

136 automatically using body attached sensors. In one such study, Weiss [41] investigated subjects walking

137 on treadmills to detect near-falls in normal activities. To generate near-fall data, different types of

138 obstacles were placed in the walking path at random. In his study, he defined near-falls similarly with trip

139 accidents. Many accelerometer-related features (e.g., signal vector magnitude, normalized signal

140 magnitude area, and other derived features) were tested in his study with an $85 \%$ sensitivity and $88 \%$

141 specificity with one feature (i.e., vertical maximum peak to peak acceleration amplitude derivative). The

142 study also showed an $85 \%$ sensitivity and $90 \%$ specificity with the combination of two features (i.e.,

143 vertical maximum peak to peak acceleration amplitude derivative and vertical maximum acceleration).

144 This study reveals the possibility of near-miss fall detection using body-attached accelerometers in a

145 controlled laboratory environment. In the construction domain, Dzeng [42] used a smartphone

146 accelerometer and gyroscope to detect fall portents (e.g., swaying, unsteady footsteps, and loss of balance)

147 which are referenced by self-reporting and video observation. To detect fall portents, Dzeng measured the

148 signal magnitude vector using accelerometer or gyroscope and detected the fall portents using a threshold-

149 based detection approach. As a result of this approach, Dzeng acquired 88.5\% accuracy for detecting fall 150 portents from construction tiling workers on a scaffold. 
152 related near misses, they predominantly focus on detecting trip or fall-portent from tiling work. Weiss [41]

153 did not consider the different types of possible loss of balance scenarios, such as kicking or stepping over

154 an obstacle, and lateral body sway in near-fall detection; additionally, the experiment in this study was

155 performed by walking on a treadmill, which sends a constant walking speed to the sensor. The fall-portent

156 detection method from Dzeng [42] tested tiling work, which deals with more stationary work movements

157 than ironworkers, whose tasks consist of diverse postures and movements such as walking, turning,

158 squatting, bolting, reaching, and carrying a load on the narrow surface of an I-beam. Moreover, the

159 detection methods from both previous studies mostly examine the magnitude of sensor signals to detect

160 near miss during controlled walking speed or stationary work conditions. Our previous study [43] which

161 used a threshold-based approach in near-miss fall detection also revealed the challenges of threshold-

162 based approaches using a single or minimal set of features. Such approaches necessitates a hierarchical

163 detection model to classify all possible variations of workers' actions. Thus, to detect near-miss falls

164 during ironworker tasks, an approach that can consider multiple features regarding body acceleration and

165 angle to differentiate near miss from dynamic movements would be more effective than using a single

166 feature. This study is extended from our earlier study [44] and proposes a semi-supervised learning

167 algorithm that enables detecting near-miss falls from dynamic movements. Upon its validation in indoor

168 and outdoor experimental settings, this study suggests its potential application to identify fall-prone

169 workers and locations in iron work.

170

\section{Methodology}

This paper aims to develop a method for automatically collecting near-miss falls among ironworkers using kinematic data acquired from WIMUs attached to workers. This study applied a semisupervised pattern-recognition algorithm (one-class support vector machine [OCSVM]) for near-miss fall detection and tested its feasibility and detection performance in a similar environment to ironworks. All computation in the study is processed through MATLAB (R2014, MATHWORKS), and OCSVM was 
176 implemented through the LIBSVM library [45], which is a popular support vector machine toolbox. The

177 OCSVM semi-supervised algorithm is a support vector machine (SVM) that is one of the most promising

178 machine learning tools for solving classification problems. Similar to the SVM algorithm, OCSVM

179 transforms the original data to a feature space and seeks the appropriate hyperplane that contains only one

180 class. Then, OCSVM uses the hyperplane as the decision boundary to classify the binary data. Two

181 different experiments were conducted to test the developed detection approach in laboratory and outdoor

182 environments. The laboratory experiments were conducted to detect ironworkers' near-miss falls to show

183 the feasibility of the developed approach. The outdoor experiments examined the performance of the

184 developed method near a construction site environment. The developed near-miss fall detection approach

185 can provide quantitative information about near misses and offers the foundations for utilizing near-miss

186 fall data to alert ironworkers about their real-time fall risks. With this approach, unrecognized near-miss

187 falls in a construction workplace can be identified, which gives researchers and safety managers the

188 chance to better understand individual workers' near-miss falls and the locations or conditions that

189 contribute to such accidents.

\subsection{Data Collection and Processing}

To test the developed near-miss fall detection method, this study conducted laboratory and

192 outdoor experiments to collect near-miss fall data on the simulated ironworks environment with five 193 subjects who participated in these experiments voluntarily. To create a test experience similar to 194 ironworkers' experiences, all experiment subjects wore safety boots, a safety harness, and a hard hat 195 during the experiments. During both experiments, each experiment subject wore a commercial WIMU 196 (Shimmer 2R, Shimmer) sensor on his/her sacrum, which is the bottom of spine and also the point nearest 197 to the human body's center of gravity. The WIMU documented the body movements of the worker 198 through a 3-axis accelerometer and gyroscope. Data from the WIMU was wirelessly transported to a 199 laptop computer in real-time using a Bluetooth connection. The overall process of the near-miss fall 200 detection appears in Figure 1 and is described in depth below. 


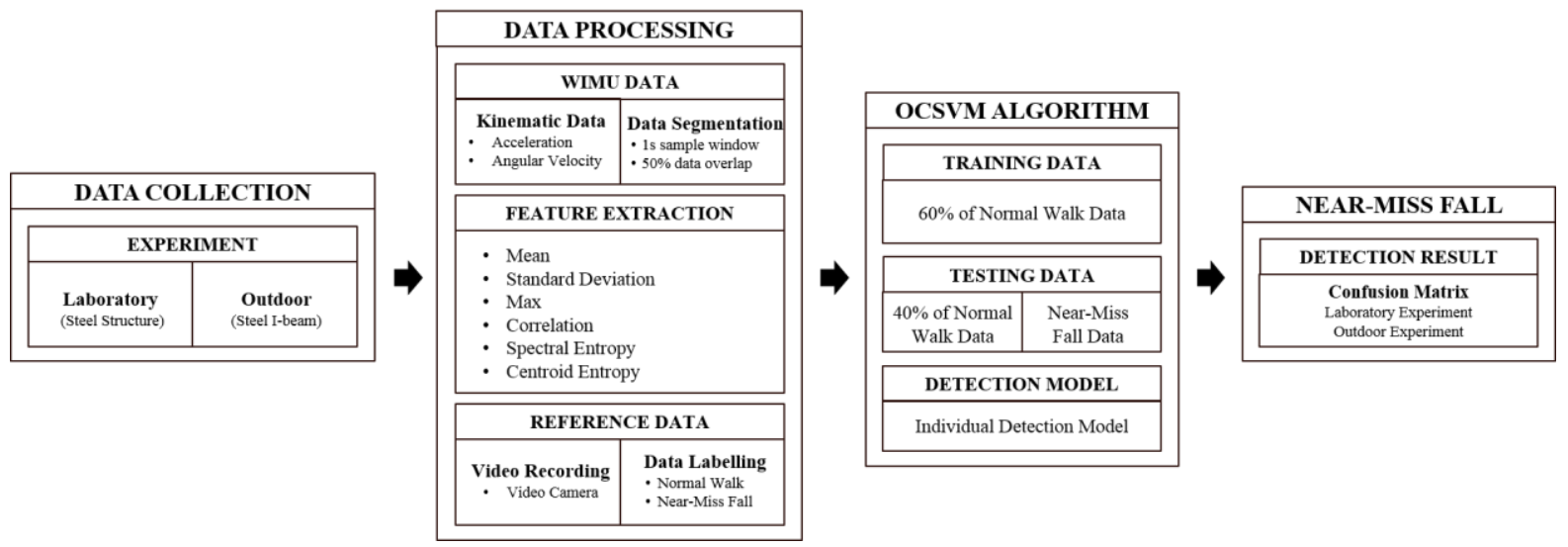




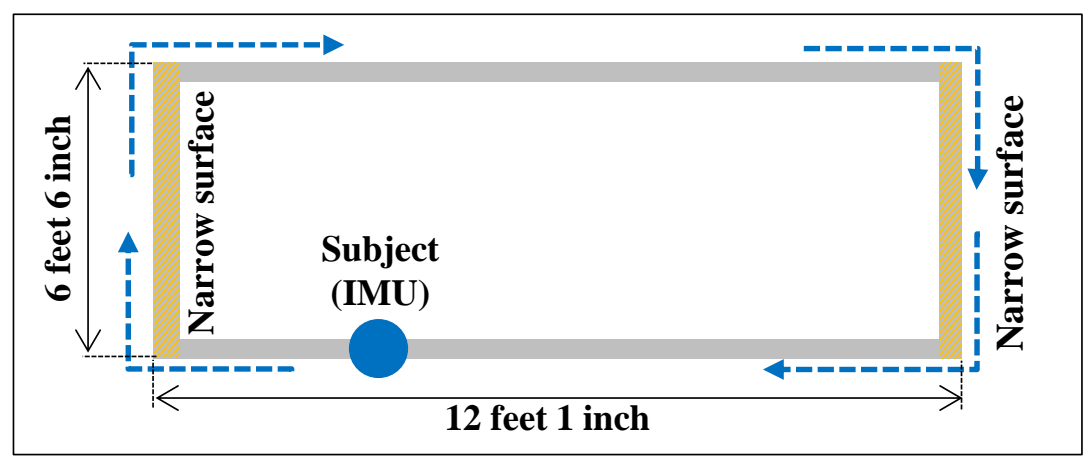

(a)

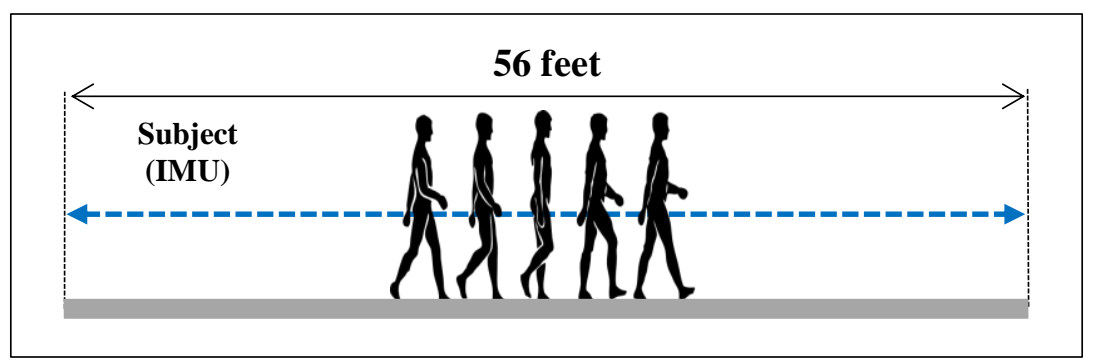

(b)

Experiment subjects were asked to walk on this steel frame without interruption over a course of

2135 minutes. During the experiment, body acceleration and angular velocity of subjects were documented 214 using a WIMU with a 51.2Hz sampling rate and the software provided by the WIMU manufacturer. In 215 addition, a video recorder filmed the experiment to document any near-miss falls so the researchers could 216 label and reference the data to identify detection results. Based on the recorded video data, the experiment 217 organizer manually labelled the near-miss falls. In the outdoor experiments, a 56' long steel I-beam with 218 an 8" width was installed in the backyard of an ironworker company. This installation was exposed to an 219 outdoor environment to simulate the working conditions of an actual construction site. As with the 220 laboratory experiments, five subjects were asked to walk continuously on the installed I-beam for 5 221 minutes. Since the I-beam installation was a single beam, the subjects were required to execute a 180 degree turn at both ends of the steel beam. 
To synchronize the video data with the WIMU data, at the beginning and end of the experiment,

224 the experiment organizer directly hit the sensor to insert a beginning signal and an ending signal into the 225 WIMU data. Although the default WIMU sampling rate is $51.2 \mathrm{~Hz}$, we reconstructed the WIMU data to use a $32 \mathrm{~Hz}$ sampling rate to ease the processing of the WIMU data because a 51.2 sampling rate mostly 227 collected data with 51 sampling rate but once with 52 sampling rate every 5 seconds. Then, using the 228 video data synchronized with the WIMU data, the experiment organizer manually labelled the occurrence 229 of near-miss falls to use the cross-reference as the ground truth in the machine learning classification. falls or stumbles - due to the diverse conditions that influence these events. Weiss [41] defined a near-fall as a loss of balance that would have resulted in a real fall if sufficient recovery process were not activated. Chehade [46] defined a stumble as a precondition of a fall that can contribute to a fall if the subject fails to recover his or her balance. Building upon these definitions, this study defines a near-miss fall as a loss of balance that results in a visible balance-recovery motion or decreased speed. Specifically, near-miss falls were labeled when experiment subjects on the I-beam had to use abnormal movements to recover their balance. Such movements included 1) Not being able to maintain the speed of walking due to a loss of balance demonstrated by having to sway the body or swing an arm, 2) Having obvious body sway or swing motions regardless of walking speed. In this study, actual falls (steps off of the beam) also occurred, 240 but their corresponding data were eliminated manually since the scope of the study did not seek to 241 classify actual falls.

To classify near-miss falls on the steel I-beam, the WIMU data were preprocessed before extracting features to reduce sensor noise and to improve the classification performance. This study used

244 a third-order Butterworth low-pass filter with a cut-off frequency of $4 \mathrm{~Hz}$ to remove sensor noise because 245 human movement energy is located below $3 \mathrm{~Hz}$ [47]. Also, this study used the 'detrend' built-in function in MATLAB (R2014, MATHWORKS) to remove the influence of gravity in the WIMU data. Then, this 247 study sampled 32 raw data to create a single data window. Using a methodology developed by a previous 
classification study [30], we then overlapped multiple adjacent data windows at their $50 \%$ mark to

249 increase the classification performance. Based upon this sample data, this study extracted 38 total features

250 in the time and frequency domains. These features are widely used in WIMU-based studies examining

251 daily activity classification and fall detection [30,48,49] and include the mean, max, standard deviation,

252 correlation, spectral entropy, and spectral centroid from the $\mathrm{x}-, \mathrm{y}-$, and $\mathrm{z}$-axis of both accelerometers and

253 gyroscopes. These 38 features were used to classify near-miss falls (unstable) and normal walking

254 motions (stable) through an OCSVM algorithm [45] (discussed in Section 3.2).

\subsection{Semi-Supervised Near-Miss Fall Detection}

Due to the various factors (e.g., missteps, trip, wind, slippery surface) at play in a construction

257 site, different types of near-miss falls can occur for the ironworker. This reality makes the use of a 258 previous threshold based fall detection algorithm [22,23] and general supervised pattern-recognition 259 algorithm challenging since such an algorithm would have to be trained with every type of near-miss fall.

260 Such a data-intensive requirement can be a huge barrier to the implementation of this method in a 261 construction site due to the difficulties in collecting all near-miss data. With the current level of 262 knowledge regarding near-miss falls — subjective definitions of near-miss falls will vary depending on 263 the perception of each individual, and what some would qualify as a near miss may differ according to the 264 ability of the subject to maintain stability or recover from a loss of balance.

265 To address this data acquisition challenge, this study examined a range of abnormal signals as stand-ins 266 for near misses. Considering that near-miss falls generates abnormal signal patterns in WIMU data, the 267 foundational premise of near-miss fall detection is the process of detecting which different types of 268 abnormal signal patterns characterize different types of near-miss falls. For example, in Figure 3, the 269 accelerometer signal for a near-miss fall (see Figure 3-c, d and e) and an actual fall (see Figure 3-b) show 270 a change in the medio-lateral axis and anterior-posterior axis acceleration as compared to normal walking 271 (Figure 3-a). 

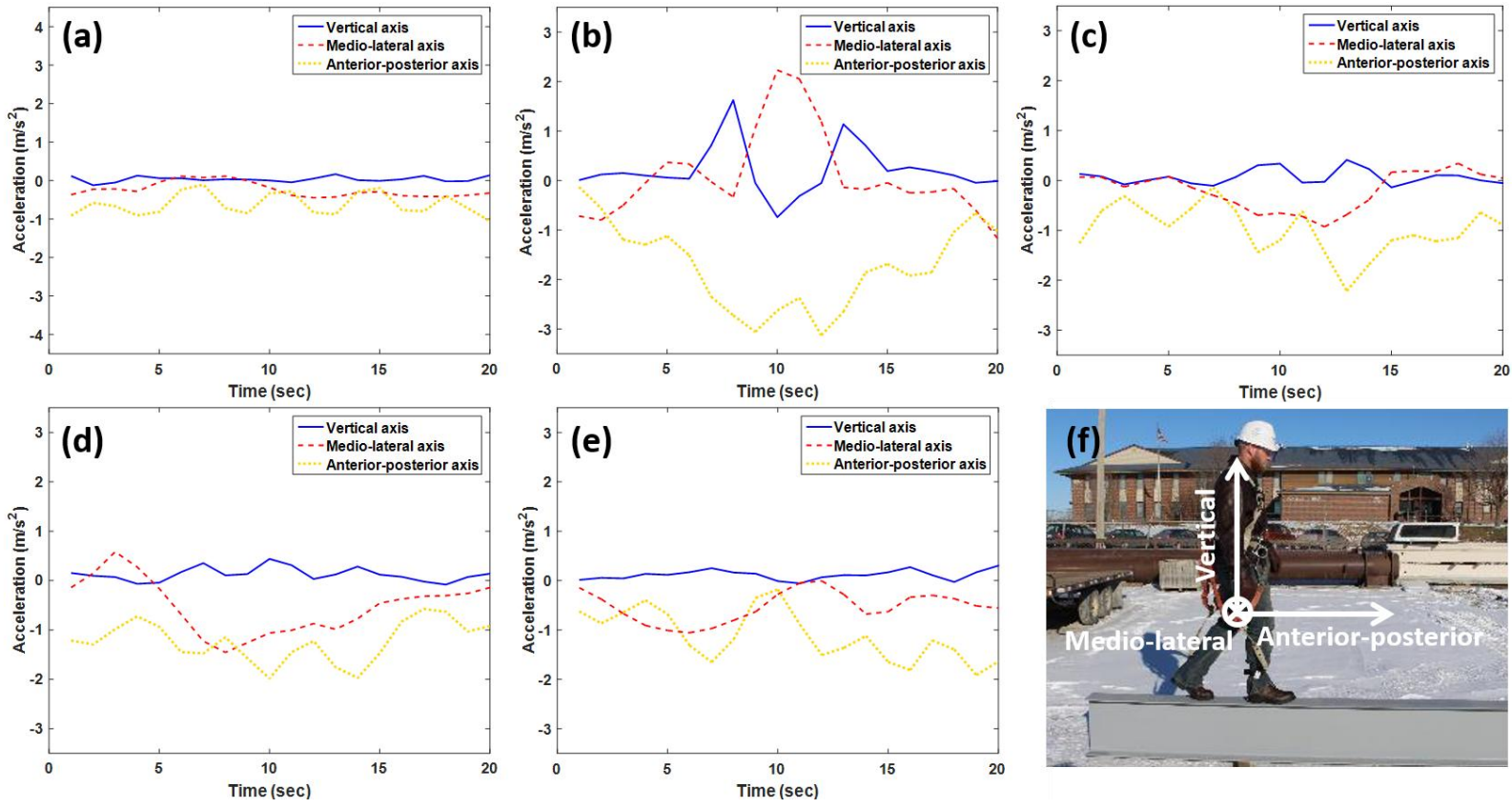

Figure 3. Average Value of Acceleration in: (a) Normal Walk, (b) Actual Fall,

However, there is a substantial difference between near-miss falls and actual falls in the acceleration of the vertical axis: As demonstrated in other fall-detection studies [22], actual falls show a high vertical acceleration whereas near-miss falls do not show a similar difference across the vertical axis and only demonstrate such substantial differences in the anterior-posterior and medio-lateral axes when compared to normal walking. Moreover, near-miss falls do not show a similar pattern to each other in terms of their acceleration across the medio-lateral and anterior-posterior axis. This characteristic of near281 miss falls makes a semi-supervised learning algorithm necessary for this study. to use an semi-supervised algorithm, one-class Support Vector Machine (OCSVM) algorithm, which can

284 detect all types of abnormal signals (near-miss falls) based upon their divergence from normal signal 285 patterns (i.e., normal walking) (see Figure 4). 

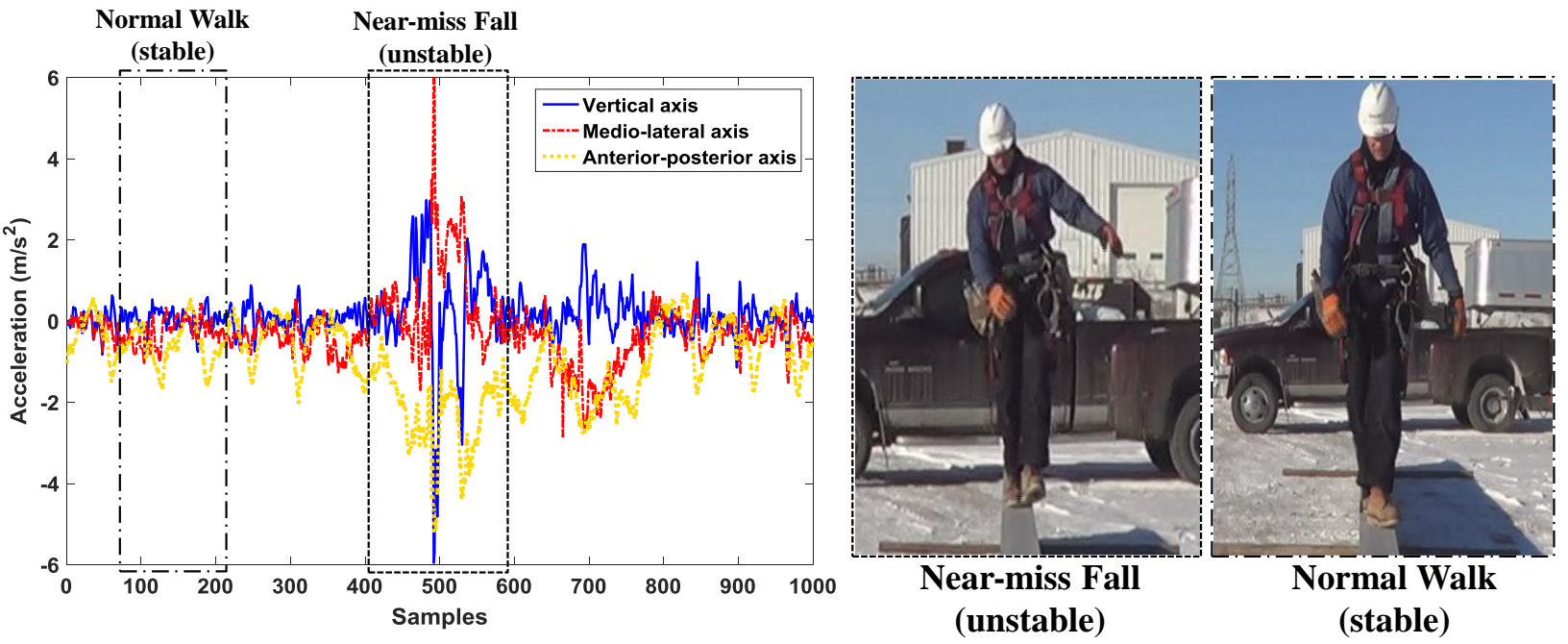

Figure 4. Concept of Near-miss Fall Detection using WIMU

According to this approach, every different near-miss fall signal does not need to be recorded and used to train the classifier. Rather, the OCSVM used in this study can classify two different classes based upon a classifier trained for only one class of data (here designated normal walking). Thus, this abnormal detection approach can be implemented by using only the normal signal data that can easily be collected in general working conditions. Instances for the algorithm are displayed by $x_{i} \in R^{n}$, and target values are $y \in\{-1,1\}^{l}$. To solve the training problem, our algorithm needed to solve this optimization problem

294 (Eq. 1) [50]:

$$
\begin{gathered}
\min _{w, \xi_{i}, \rho} \frac{1}{2}\|w\|^{2}+\frac{1}{v n} \sum_{i=1}^{n} \xi_{i}-\rho \\
\text { Subject to }\left(w^{t} \phi\left(x_{i}\right)\right) \geq \rho-\xi_{i} \text { and } \xi_{i} \geq 0 \\
\mathrm{f}(\mathrm{x})=\operatorname{sign}\left(\left(w \cdot \phi\left(x_{i}\right)-\rho\right)\right.
\end{gathered}
$$

Here, $v$ is a introduced parameter between 0 and 1 which is an upper limit on the fraction of training errors and lower limit of the fraction of support vector [51], and $\xi_{i}$ is a slack variable measuring the error of $x_{i}$. 
separates the classes. When solved optimization problem with $\mathrm{w}$ and $\rho$, class will be decided using

299 decision function (Eq. 2). In this study, various kernels have been studied, and the best results were 300 achieved using a radial basis function $(\mathrm{RBF})$ :

$$
\begin{gathered}
\mathrm{K}\left(X_{i}, X_{j}\right)=\exp \left(-r\left\|X_{i}-X_{j}\right\|^{2}\right), r>0 \\
\mathrm{~K}\left(X_{i}, X_{j}\right)=\left(\phi\left(x_{i}\right) \cdot \phi\left(x_{j}\right)\right)
\end{gathered}
$$

An RBF kernel is widely used to handle the nonlinear classification problem when the relationship between the target values and the attributes is nonlinear [52]. With this kernel, all kernel values sit in the range $[0,1]$, which results in a simplicity of computing vectors rather than polynomial

304 kernels that can yield infinite values [53]. An RBF kernel needs to define the parameter $(r)$ to perform 305 better in training classification performance. To achieve the best values, we performed a grid search by 306 exponentially increasing the parameter. Once an approximate value for each parameter was found via a 307 coarse grid, we narrowed down the search using a finer grid. Then, we selected the parameter value that 308 offered the best accuracy in near-miss fall detection. When it came to training the algorithm, all training

309 data were selected from normal walking data that did not include a near-miss fall. In this study, $60 \%$ of 310 the normal walking data was used for training the OCSVM classifier, and then the rest of the normal 311 walking as well as the near-miss fall data were used to test the trained classifier. . During the 312 classification process, we adjusted the parameters of the algorithm to attempt to improve an accuracy of 313 our algorithm.

\section{4. Results}

This study subsequently assessed the accuracy of the overall near-miss fall detection approach by producing a confusion matrix (see Table 1). According to the two different experiment conditions (i.e.,

317 the laboratory experiment and the outdoor experiment) and the outcomes of the five subjects, our 318 resulting algorithm detected near-miss fall occurrences with $75.8 \%$ recall (TP / $(\mathrm{TP}+\mathrm{FN})$ ) and $86.4 \%$ 
overall detection accuracy $((\mathrm{TP}+\mathrm{TN}) /(\mathrm{P}+\mathrm{N}))$. During the laboratory experiment, the test subjects

320 experienced a cumulative 183 near-miss falls on the installed steel frame. Among these near-miss falls,

321137 data samples were correctly classified (74.9\%) using the algorithm. In the outdoor experiments, a

322 total of 69 near-miss falls occurred, and 54 of them were successfully detected (78.3\%) through the

323 implemented algorithm.

To foster near-misses in the data samples, this study installed narrow surface beams in the laboratory experiment. As a result, more near-miss falls (183 samples) occurred compared to the outdoor experiments (69 samples) and it is expected to link to the better detection results in near-miss fall detection. However, in spite of larger number of near-miss data, laboratory experiment shows slightly

328 lower recall compared to outdoor experiment. In fact, the narrow surface beams in the laboratory 329 experiments presented a difficulty for subjects as they tried to perform their normal walking movements.

330 Their walking movements thus included other simultaneous motions such as extensive arm swing,

331 inconstant walking speed, and irregular balance-recovery motions. These motions caused ambiguity in

332 labelling normal walking in the laboratory experiments. On the other hand, the outdoor experiments faced

333 less ambiguity in the labeling of the normal walking due to simplicity of experiment layout (i.e. wider

334 walking surface). This detail explains the slightly lower recall in the laboratory experiments (74.9\%) as 335 compared to the outdoor experiments (78.3\%).

Table 1. Near-Miss Fall Detection Results Achieved Through the OCSVM

\begin{tabular}{|c|c|c|c|c|c|c|}
\hline \multirow[b]{2}{*}{ Activity } & \multirow{2}{*}{$\begin{array}{c}\text { Subject } \\
\#\end{array}$} & \multirow{2}{*}{$\begin{array}{c}\text { Number of } \\
\text { Occurred } \\
\text { Near-miss } \\
\text { falls }\end{array}$} & \multirow{2}{*}{$\begin{array}{c}\text { Number of } \\
\text { Detected } \\
\text { Near-miss } \\
\text { falls }\end{array}$} & \multicolumn{3}{|c|}{ Confusion Matrix } \\
\hline & & & & & $\begin{array}{l}\text { Near miss } \\
\text { (Predicted) }\end{array}$ & $\begin{array}{c}\text { Walk } \\
\text { (Predicted) }\end{array}$ \\
\hline \multirow{7}{*}{$\begin{array}{c}\text { Laborat } \\
\text { ory }\end{array}$} & \multirow{2}{*}{1} & \multirow{2}{*}{58} & \multirow{2}{*}{45} & Near miss & $77.6 \%$ & $22.4 \%$ \\
\hline & & & & Walk & $9.7 \%$ & $90.3 \%$ \\
\hline & \multirow{2}{*}{2} & \multirow{2}{*}{25} & \multirow{2}{*}{18} & Near miss & $72 \%$ & $28 \% \%$ \\
\hline & & & & Walk & $13.4 \%$ & $86.6 \%$ \\
\hline & \multirow{2}{*}{3} & \multirow{2}{*}{46} & \multirow{2}{*}{35} & Near miss & $76.1 \%$ & $23.9 \%$ \\
\hline & & & & Walk & $16.4 \%$ & $83.6 \%$ \\
\hline & 4 & 22 & 16 & Near miss & $72.7 \%$ & $27.3 \%$ \\
\hline
\end{tabular}




\begin{tabular}{|c|c|c|c|c|c|c|}
\hline & & & & Walk & $11 \%$ & $88.9 \%$ \\
\hline & \multirow{2}{*}{5} & \multirow{2}{*}{32} & \multirow{2}{*}{23} & Near miss & $71.9 \%$ & $14.4 \%$ \\
\hline & & & & Walk & $10 \%$ & $90 \%$ \\
\hline & \multirow{2}{*}{ Total } & \multirow{2}{*}{183} & \multirow{2}{*}{137} & Recall & \multicolumn{2}{|c|}{$74.9 \%$} \\
\hline & & & & Accuracy & \multicolumn{2}{|c|}{$86.8 \%$} \\
\hline \multirow{12}{*}{$\begin{array}{l}\text { Outdoo } \\
\quad \mathrm{r}\end{array}$} & \multirow{2}{*}{1} & \multirow{2}{*}{28} & \multirow{2}{*}{22} & Near miss & $78.6 \%$ & $21.4 \%$ \\
\hline & & & & Walk & $15.7 \%$ & $84.3 \%$ \\
\hline & \multirow{2}{*}{2} & \multirow{2}{*}{7} & \multirow{2}{*}{6} & Near miss & $85.7 \%$ & 14.3 \\
\hline & & & & Walk & $14.3 \%$ & $85.7 \%$ \\
\hline & \multirow{2}{*}{3} & \multirow{2}{*}{12} & \multirow{2}{*}{9} & Near miss & $75 \%$ & $25 \%$ \\
\hline & & & & Walk & $12.1 \%$ & $87.9 \%$ \\
\hline & \multirow{2}{*}{4} & \multirow{2}{*}{5} & \multirow{2}{*}{4} & Near miss & $80 \%$ & $20 \%$ \\
\hline & & & & Walk & $11.8 \%$ & $88.2 \%$ \\
\hline & \multirow{2}{*}{5} & \multirow{2}{*}{17} & \multirow{2}{*}{13} & Near miss & $76.5 \%$ & $23.5 \%$ \\
\hline & & & & Walk & $16.3 \%$ & $83.7 \%$ \\
\hline & \multirow{2}{*}{ Total } & \multirow{2}{*}{69} & \multirow{2}{*}{54} & Recall & \multicolumn{2}{|c|}{$78.3 \%$} \\
\hline & & & & Accuracy & & \\
\hline
\end{tabular}

For walking activity classification, the developed approach achieved $86.4 \%$ recall in the two

340 experiments, and the remaining $14.6 \%$ of walking data were misclassified as near-miss falls. The

341 misclassified walking incidents occurred near the edge of the steel beam before/after subjects made a

342 turning motion. At this point, the subjects' walk speed decreased as they prepared for the turn, or they

343 started to walk, which may have given a similar signal to the near-miss fall defined in this study (i.e.,

344 when subjects reduced their walk speed and recovered their balance with an arm swing without

345 significant body sway). Also, an irregular walk, such as wide/small step distance, steps with strong

346 vertical force, and small degrees of body motion, were sometimes misclassified as near-miss falls using

347 the developed approach. This result shows that irregular walking patterns, especially irregular steps and

348 small body motions, have a similar signal pattern as defined near-miss falls when using a single WIMU

349 (attached to the sacrum). This result illustrates that multiple WIMUs (e.g., attached to the head or upper

350 body) may be necessary to better classify subtle movements. Also, a better definition or representation of

351 near-miss falls would be beneficial to clearly differentiate between near-miss falls and irregular walking 352 movements. 
In the laboratory experiments, near-miss falls were detected with similar accuracy $(71.9 \%$ to

$35477.6 \%$ ) across experiment subjects. However, outdoor experiments had a varied near-miss fall detection 355 rate $(73 \%$ to $87.5 \%)$ depending on the experiment subjects. Such a high variability of near-miss fall 356 detection rates manifested in-part due to the low incidences of near-miss falls in the outdoor experiment 357 setting. For example, Subjects 2 and 5 had fewer near-miss falls compared to Subjects 1, 3, and 5 during 358 the outdoor experiments, and the near-miss fall detection rates in Subjects 2 and 5 were found to be 359 higher than other subjects. It should be noted that Subject 2 and 5 also had fewer near-miss falls even in 360 the laboratory experiments. Such a consistency of individual vulnerability to near-miss falls across 361 different experimental settings indicates that the frequency of detected near-miss falls could be used as a 362 predictor of the fall risks of individual workers.

\section{Discussion}

As a proactive fall accident-prevention measure for ironworker, this study developed a near-miss

366 fall detection approach that uses wearable inertial measurement units (WIMUs) to gather data about 367 bodily motions. The data are then be applied to an algorithm that monitors bodily gestures for abnormal movements (near misses). In particular, this study successfully used WIMUs to document the signals of subjects losing their balance (near-miss falls) and detected these signal using a well-known abnormality detection algorithm (OCSVM). To verify the developed near-miss fall detection approach, this study conducted two different experiments in laboratory and outdoor settings. By applying the OCSVM algorithm, this study achieved moderate near-miss fall detection accuracies regardless of the experimental environment.

Previous studies to detect a near-miss fall based on WIMU data mostly used a threshold approach based on the sum of total body acceleration [30,31]. While such studies demonstrated the feasibility of their approach in limited settings (e.g., during treadmill walking, stationary motions), the threshold approach requires collecting and analyzing all possible forms of near-miss fall data and normal activity 
data to define the threshold values of near-miss falls. This task may not be feasible considering the nature of near misses in construction. The proposed approach based on the OCSVM algorithm is able to detect different types of near-miss falls based on workers' normal activity data, which is relatively easy to obtain. In addition, unlike previous studies, which focused on detecting trip/ slip or detecting near-miss fall

382 during stationary actions, this study is an initial attempt at detecting near-miss falls, loss of balance, 383 during non-stationary actions (i.e., moving along the steel frame). Such non-stationary actions included 384 more diverse motions (e.g., turning at the edge of steel frames, acceleration and deceleration of walking 385 speed), and irregular movements of other body parts (e.g., extensive arm swings), all of which posed a 386 greater challenge in detecting near-miss falls and would explain the slightly lower accuracy of the 387 proposed approach compared to previous studies. fruitful point of discussion. By synthesizing the detected near-miss fall information for multiple subjects walking along the steel beams, the locations that appear to contribute to making workers fall-prone can be 391 identified (see Figure 5). To document the locations of the collected near-miss falls on the steel structure, 392 each turning motion was labeled with video data. Then, the near-miss fall data were identified as before or after the turning motion and the total number of data samples between each turning motion were

394 resized to have same length for each steel beam. As a result, the narrow surface beams-marked as A 395 and $\mathrm{C}$ - show relatively higher occurrences of near-miss falls even when utilizing data from only one 396 subject. With the current accuracy of the OCSVM algorithm, the figure shows a clear difference between 397 the wide surfaces and the narrow surfaces in the laboratory experiment. This outcome indicates that when 398 synthesizing the near-miss fall data of multiple workers in a particular location, this fall-prevention 399 approach could help identify hazardous conditions automatically. Thus, by combining the localization 400 techniques, this near-miss fall detection approach has the high potential to detect hazardous conditions 401 and to contribute to reducing the risk of fall accidents by decreasing the exposure time workers face 402 hazards detected within the collected locational information. 

417 of construction job tasks.

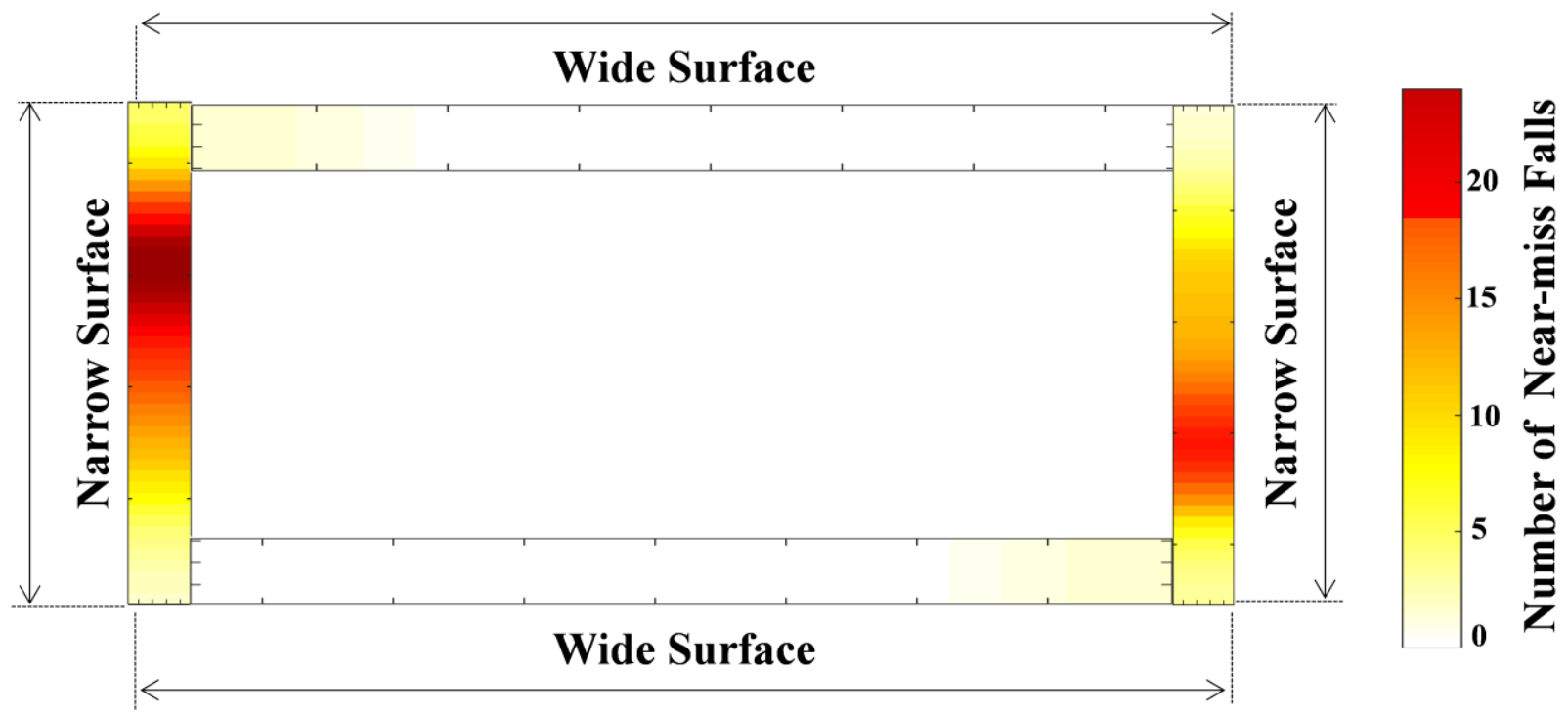

Figure 5. Near-Miss Fall Locations in Laboratory Setting

However, there are still many challenges to addressing the diversity of construction job tasks. For example, carrying a symmetrical or asymmetrical load may affect to the IMU signals of normal walking and near-miss falls. In response, this study also tested the detection performance of the algorithm for a case in which subjects carried a side-load (25lb). All other experimental setting were maintained exactly as those of the previous outdoor experiments. Though this new experiment included an asymmetrical load, we first trained the OCSVM classifier using normal walking data collected from the outdoor experiments in which the subjects carried no loads - this choice allowed us to examine the importance of building out a comprehensive training dataset. Our results showed that when we trained the classifier using the no-load data, the recall of near-miss detection dropped by $18 \%$ compared to previous outdoor experiments. However, when the classifier was trained with data collected from the walking with a side-load experiment, its recall accuracy proved to be slightly better than previous outdoor experiments. This preliminary result indicates the need for building a comprehensive training dataset to address the diversity

An additional consideration that expands the need for a robust and automated approach to fall accident-prevention has to do with the variability of workers' balance. For example, different people have 
different abilities to maintain their balance while working on a small surface area (specifically working on

421 a narrow steel beam). Furthermore, even an individual worker may experience variability in his/her

422 abilities to maintain a balance depending on his/her workload or fatigue level. Some types of hazards-

423 such as slippery surfaces, obstacles on the beam, strong wind, or uneven or moving surfaces—can also

424 impact workers' balance, but such hazards are often ignored in current hazard identification processes.

425 Collecting and analyzing near-miss fall data using this proposed approach will help identify workers who

426 are fall-prone due to excessive workload or fatigue and/or will help locate hidden fall hazards that are not

427 identified by current hazard analysis practices.

\section{Conclusion}

This study developed and tested an approach to detect the near-miss falls of ironworkers based on WIMU data attached to workers' bodies. An OCSVM algorithm was implemented to detect the near-miss falls based upon a binary assessment of the normal walking movements. The results demonstrated the performance of this approach by achieving a $75.8 \%$ recall and $87.5 \%$ accuracy in detecting near-miss falls with two different experimental conditions that simulate ironworker working environments.

This study validated the feasibility of the developed approach in detecting near-miss falls and demonstrated its usefulness as a proactive fall-prevention method for ironworkers. With the advantage of a semi-supervised algorithm that classifies near-miss falls using only normal walking data, the developed

437 approach has substantial benefits in real-world implementation compared to previous approaches. The 438 developed approach will contribute to providing near-miss fall information that can be used for proactive 439 fall-prevention measures of ironworkers in response to their high risk of fall accidents. Also, this 440 approach is expected to help safety managers identify individual fall risks by counting the total number of 441 near-miss falls for each worker and predicting risky locations or hazards based upon the detected 442 locations of near-miss falls. Specifically, collected near-miss fall locations would beneficial for analyzing 443 the relationship between ironworkers' behavior patterns and the construction site environment. The 
444 proposed WIMU-based approach also has application and financial benefits due to the small sensor size 445 and the low operation costs.

As a limitation to the approach, the near-miss fall detection accuracy varies depending upon the 447 experiment subject and the performed task. Further studies on near-miss fall detection during diverse 448 ironworker activities, including squatting, bolting, reaching, and carrying a load, need to be conducted to 449 improve the applicability of this approach. Also, additional field experiments in actual building structures 450 are necessary to validate the effectiveness of the developed approach in identifying potential problems 451 (e.g., hazards) in a real-world setting. Finally, future studies should test the applicability of this falldetection approach in different construction trades to expand the reach of this study.

\section{Acknowledgement}

The authors would like to acknowledge the excellent support and technical assistance from Cory conducting the experiment. This study was financially supported by Nebraska Research Initiative and National Science Foundation (CMMI \#1538029 and CNS \#1423379). Any opinions, findings, conclusions, or recommendations expressed in this article are those of the authors and do not necessarily

459 reflect the views of Nebraska Research Initiative and National Science Foundation.

\section{Reference}

462 [1] BLS, Bureau of Labor Statistics, United States Department of Labor, 2013.

463 [2] CPWR, The Construction Chart Book, The Center for Construction Research and Training, 2013.

464 [3] J. Beavers, J. Moore, W. Schriver, Steel Erection Fatalities in the Construction Industry, Journal of 465 Construction Engineering and Management. 135 (2009) 227-234. doi:10.1061/(ASCE)07339364(2009)135:3(227). 
[4] C.-F. Chi, T.-C. Chang, H.-I. Ting, Accident patterns and prevention measures for fatal occupational falls in the construction industry, Applied Ergonomics. 36 (2005) 391-400. doi:10.1016/j.apergo.2004.09.011.

[5] T.G. Bobick, R.L. Stanevich, T.J. Pizatella, P.R. Keane, D.L. Smith, Preventing falls through

[6] H. Hsiao, P. Simeonov, Preventing falls from roofs: a critical review, Ergonomics. 44 (2001) 537skylights and roof openings, Professional Safety. 39 (1994) 33.

474 [7] C. Lee, K.M. Porter, Suspension trauma, Emergency Medicine Journal. 24 (2007) 237-238. doi:10.1136/emj.2007.046391.

[8] X. Huang, J. Hinze, Analysis of Construction Worker Fall Accidents, Journal of Construction Engineering and Management. 129 (2003) 262-271. doi:10.1061/(ASCE)0733-

[9] M. Grabowski, P. Ayyalasomayajula, J. Merrick, D. Mccafferty, Accident precursors and safety nets: leading indicators of tanker operations safety, Maritime Policy \& Management. 34 (2007) 405-425. doi:10.1080/03088830701585084.

[10] J. Toellner, Improving safety and health performance: Identifying and measuring leading indicators, Professional Safety. 46 (2001) 42-47.

484

[11] J. Hinze, S. Thurman, A. Wehle, Leading indicators of construction safety performance, Safety Science. 51 (2013) 23-28. doi:10.1016/j.ssci.2012.05.016.

[12] W. Wu, A.G.F. Gibb, Q. Li, Accident precursors and near misses on construction sites: An investigative tool to derive information from accident databases, Safety Science. 48 (2010) 845-858. doi:10.1016/j.ssci.2010.04.009.

[13] V.M. Bier, A. Mosleh, The analysis of accident precursors and near misses: Implications for risk assessment and risk management, Reliability Engineering \& System Safety. 27 (1990) 91-101. doi:10.1016/0951-8320(90)90033-J. 
[14] J.R. Phimister, U. Oktem, P.R. Kleindorfer, H. Kunreuther, Near-Miss Incident Management in the Chemical Process Industry, Risk Analysis. 23 (2003) 445-459. doi:10.1111/1539-6924.00326.

[15] L. Wright, T. van der Schaaf, Accident versus near miss causation: a critical review of the literature, an empirical test in the UK railway domain, and their implications for other sectors, Journal of Hazardous Materials. 111 (2004) 105-110. doi:10.1016/j.jhazmat.2004.02.049.

[16] C.H. Tinsley, R.L. Dillon, M.A. Cronin, How Near-Miss Events Amplify or Attenuate Risky Decision Making, Management Science. 58 (2012) 1596-1613. doi:10.1287/mnsc.1120.1517.

[17] M.G. Gnoni, G. Lettera, Near-miss management systems: A methodological comparison, Journal of Loss Prevention in the Process Industries. 25 (2012) 609-616. doi:10.1016/j.jlp.2012.01.005.

[18] T. van der Schaaf, L. Kanse, Biases in incident reporting databases: an empirical study in the chemical process industry, Safety Science. 42 (2004) 57-67. doi:10.1016/S0925-7535(03)00023-7.

[19] F.B. Cambraia, T.A. Saurin, C.T. Formoso, Identification, analysis and dissemination of information on near misses: A case study in the construction industry, Safety Science. 48 (2010) 91-99. doi:10.1016/j.ssci.2009.06.006.

[20] Z. Zhou, J. Irizarry, Q. Li, W. Wu, Using Grounded Theory Methodology to Explore the Information of Precursors Based on Subway Construction Incidents, Journal of Management in Engineering. 31 (2013). doi:10.1061/(ASCE)ME.1943-5479.0000226.

[21] W. Wu, H. Yang, D.A.S. Chew, S. Yang, A.G.F. Gibb, Q. Li, Towards an autonomous real-time tracking system of near-miss accidents on construction sites, Automation in Construction. 19 (2010) 134-141. doi:10.1016/j.autcon.2009.11.017.

[22] M. Kangas, A. Konttila, P. Lindgren, I. Winblad, T. Jämsä, Comparison of low-complexity fall detection algorithms for body attached accelerometers, Gait Posture. 28 (2008) 285-291. doi:10.1016/j.gaitpost.2008.01.003.

[23] A.K. Bourke, G.M. Lyons, A threshold-based fall-detection algorithm using a bi-axial gyroscope sensor, Medical Engineering \& Physics. 30 (2008) 84-90. doi:10.1016/j.medengphy.2006.12.001. 
[24] C.-F. Lai, S.-Y. Chang, H.-C. Chao, Y.-M. Huang, Detection of Cognitive Injured Body Region Using Multiple Triaxial Accelerometers for Elderly Falling, IEEE Sensors Journal. 11 (2011) 763770. doi:10.1109/JSEN.2010.2062501.

[25] J. Liu, X. Zhang, T.E. Lockhart, Fall Risk Assessments Based on Postural and Dynamic Stability Using Inertial Measurement Unit, Safety and Health at Work. 3 (2012) 192-198. doi:10.5491/SHAW.2012.3.3.192.

[26] N. Pannurat, S. Thiemjarus, E. Nantajeewarawat, Automatic Fall Monitoring: A Review, Sensors. 14 (2014) 12900-12936. doi:10.3390/s140712900.

[27] S.J. Preece, J.Y. Goulermas, L.P.J. Kenney, D. Howard, K. Meijer, R. Crompton, Activity identification using body-mounted sensors - a review of classification techniques, Physiological Measurement. 30 (2009) R1. doi:10.1088/0967-3334/30/4/R01.

[28] X. Long, B. Yin, R.M. Aarts, Single-accelerometer-based daily physical activity classification, Annual International Conference of the IEEE Engineering in Medicine and Biology Society, 2009. EMBC 2009, 2009: pp. 6107-6110. doi:10.1109/IEMBS.2009.5334925.

[29] A. Godfrey, A.K. Bourke, G.M. Ólaighin, P. van de Ven, J. Nelson, Activity classification using a single chest mounted tri-axial accelerometer, Medical Engineering \& Physics. 33 (2011) 1127-1135. doi:10.1016/j.medengphy.2011.05.002.

[30] S. Chernbumroong, A.S. Atkins, H. Yu, Activity classification using a single wrist-worn accelerometer, in: 2011 5th International Conference on Software, Knowledge Information, Industrial Management and Applications (SKIMA), 2011: pp. 1-6. doi:10.1109/SKIMA.2011.6089975.

[31] S.D. Bersch, D. Azzi, R. Khusainov, I.E. Achumba, J. Ries, Sensor data acquisition and processing parameters for human activity classification, Sensors. 14 (2014) 4239-4270. doi:10.3390/s140304239

[32] W.P. Bennett, M. Fitzpatrick, D. Anthony, M.C. Vuran, A. Lacy, Crane Charades: Behavior Identification via Backpack Mounted Sensor Platforms, in: Proceedings of the 11th International 
Conference on Information Processing in Sensor Networks, ACM, New York, NY, USA, 2012: pp. 119-120. doi:10.1145/2185677.2185706.

[33] D. Anthony, W.P. Bennett, M.C. Vuran, M.B. Dwyer, S. Elbaum, A. Lacy, et al., Sensing through the continent: towards monitoring migratory birds using cellular sensor networks, in: Proceedings of the 11th international conference on Information Processing in Sensor Networks, ACM, New York, NY, USA, 2012: pp. 329-340. doi:10.1145/2185677.2185747.

[34] S. Yang, J.-T. Zhang, A.C. Novak, B. Brouwer, Q. Li, Estimation of spatio-temporal parameters for post-stroke hemiparetic gait using inertial sensors, Gait \& Posture. 37 (2013) 354-358. doi:10.1016/j.gaitpost.2012.07.032.

[35] A. Muro-de-la-Herran, B. Garcia-Zapirain, A. Mendez-Zorrilla, Gait Analysis Methods: An Overview of Wearable and Non-Wearable Systems, Highlighting Clinical Applications, Sensors. 14 (2014) 3362-3394. doi:10.3390/s140203362.

[36] R. Moe-Nilssen, A new method for evaluating motor control in gait under real-life environmental conditions. Part 1: The instrument, Clinical Biomechanics. 13 (1998) 320-327. doi:10.1016/S02680033(98)00089-8.

[37] G.H. Cattledge, A. Schneiderman, R. Stanevich, S. Hendricks, J. Greenwood, Nonfatal occupational fall injuries in the West Virginia construction industry, Accident Analysis \& Prevention. 28 (1996) 655-663. doi:10.1016/0001-4575(96)00026-7.

[38] R. Navon, O. Kolton, Model for Automated Monitoring of Fall Hazards in Building Construction, Journal of Construction Engineering and Management. 132 (2006) 733-740. doi:10.1061/(ASCE)0733-9364(2006)132:7(733).

[39] M.J. Skibniewski, Information technology applications in construction safety assurance, Journal of Civil Engineering and Management. 20 (2014) 778-794. doi:10.3846/13923730.2014.987693.

[40] A.A. Ahmed, J.A. Ashton-Miller, Is a "loss of balance" a control error signal anomaly? Evidence for three-sigma failure detection in young adults, Gait \& Posture. 19 (2004) 252-262. doi:10.1016/S0966-6362(03)00066-3. 
[41] A. Weiss, I. Shimkin, N. Giladi, J.M. Hausdorff, Automated detection of near falls: algorithm development and preliminary results, BMC Research Notes. 3 (2010) 62. doi:10.1186/1756-0500-362.

[42] R.-J. Dzeng, Y.-C. Fang, I.-C. Chen, A feasibility study of using smartphone built-in accelerometers to detect fall portents, Automation in Construction. 38 (2014) 74-86. doi:10.1016/j.autcon.2013.11.004.

[43] K. Yang, H. Jebelli, C.R. Ahn, M.C. Vuran, Threshold-Based Approach to Detect Near-Miss Falls of Iron Workers Using Inertial Measurement Units, in: Computing in Civil Engineering 2015, American Society of Civil Engineers, 2015: pp. 148-155. doi:10.1061/9780784479247.019.

[44] K. Yang, S. Aria, T.L. Stentz, C.R. Ahn, Automated Detection of Near-miss Fall Incidents in Iron Workers Using Inertial Measurement Units, in: Construction Research Congress 2014, American Society of Civil Engineers, 2014: pp. 935-944. doi:10.1061/9780784413517.096.

[45] C.-C. Chang, C.-J. Lin, LIBSVM: A Library for Support Vector Machines, ACM Transactions on Intelligent Systems and Technology (TIST). 2 (2011) 27:1-27:27. doi:10.1145/1961189.1961199.

[46] N.H. Chehade, P. Ozisik, J. Gomez, F. Ramos, G. Pottie, Detecting stumbles with a single accelerometer, in: 2012 Annual International Conference of the IEEE Engineering in Medicine and Biology Society (EMBC), 2012: pp. 6681-6686. doi:10.1109/EMBC.2012.6347527.

[47] W. Wang, Y.-W. Guo, B.-Y. Huang, G.-R. Zhao, B. Liu, L. Wang, Analysis of filtering methods for 3D acceleration signals in body sensor network, in: 2011 International Symposium on Bioelectronics and Bioinformatics (ISBB), 2011: pp. 263-266. doi:10.1109/ISBB.2011.6107697.

[48] M. Zhang, A.A. Sawchuk, A Feature Selection-based Framework for Human Activity Recognition Using Wearable Multimodal Sensors, in: Proceedings of the 6th International Conference on Body Area Networks, ICST (Institute for Computer Sciences, Social-Informatics and Telecommunications Engineering), ICST, Brussels, Belgium, Belgium, 2011: pp. 92-98. http://dl.acm.org/citation.cfm?id=2318776.2318798 (accessed April 7, 2015). 
594 [49] A. Bulling, U. Blanke, B. Schiele, A tutorial on human activity recognition using body-worn inertial sensors, ACM Computing Surveys (CSUR). 46 (2014) 33. doi:10.1145/2499621

596 [50] L.M. Manevitz, M. Yousef, One-class SVMs for document classification, The Journal of Machine 597 Learning Research. 2 (2002) 139-154. doi:10.1023/A:1007369909943

598 [51] B. Schölkopf, J.C. Platt, J. Shawe-Taylor, A.J. Smola, R.C. Williamson, Estimating the Support of a 599 High-Dimensional Distribution, Neural Computation. 13 (2001) 1443-1471.

$600 \quad$ doi:10.1162/089976601750264965.

601 [52] M. Girolami, Mercer kernel-based clustering in feature space, IEEE Transactions on Neural $602 \quad$ Networks. 13 (2002) 780-784. doi:10.1109/TNN.2002.1000150.

603 [53] C.-J. Lin, Formulations of support vector machines: a note from an optimization point of view, $604 \quad$ Neural Computation. 13 (2001) 307-317. doi:10.1162/089976601300014547 